\title{
Simplicial normalization in the entire cyclic cohomology of Banach algebras
}

\author{
Jacek Brodzki \\ Department of Mathematical Sciences \\ University of Durham \\ South Road, Durham DH1 3LE, England
}

\begin{abstract}
We show that the entire cyclic cohomology of Banach algebras defined by Connes has the simplicial normalization property. A key tool in the proof is the notion and properties of supertraces on the Cuntz algebra $Q A$. As an example of further applications of this technique we give a proof of the homotopy invariance of entire cyclic cohomology.
\end{abstract}

Key words Simplicial normalization, homotopy invariance, supertraces, Cuntz algebra, entire cyclic cohomology.

\section{Introduction}

One of the basic features of the non-commutative geometry introduced in the fundamental work of Connes [Co1, Co3] is that in order to have a good analogue of the de Rham homology for non-commutative spaces one needs to introduce cyclic cohomology, or more generally, theories of cyclic type, like periodic cyclic cohomology. In particular, the periodic cyclic cohomology is the natural target for the Chern character map from $K$-homology. In the 'finite dimensional' situation, the $K$-cycles are described by means of $p$-summable Fredholm modules, which are determined by a representation of an algebra $A$ in a Hilbert space $H$ together with an involution $F$, acting on $H$, such that $[F, a] \in \mathcal{L}^{p}(H)$, for all $a \in A$, where $\mathcal{L}^{p}(H)$ is the $p$-th Schatten ideal in the algebra of bounded operators on $H$. There are examples that demonstrate that in certain situations the hypothesis of finite summability should be replaced by a weaker condition. The new condition of $\theta$-summability [Co2] is reflected on cyclic cohomology by introduction of a growth condition on the cochains, which are now elements of a certain $\mathbf{Z} / 2$-graded complex, the entire cyclic cochain complex.

We define the entire cochain complex as follows. A cochain in this complex is a possibly infinite sequence of multilinear functionals on $A$

$$
\left(f_{0}, f_{1}, \ldots, f_{n}, \ldots\right)
$$

where $f_{n}: A^{\otimes n+1} \rightarrow \mathbf{C}$. A cochain is called even (odd) if its components $f_{n}$ are nonzero when $n$ is even (odd). This way we obtain a $\mathbf{Z} / 2$-graded complex equipped

\footnotetext{
${ }^{0}$ To apperar in $K$-theory
} 
with the differential $b+B$ acting between spaces of cochains of opposite parity. This complex is not very interesting as its cohomology is trivial. When $A$ is a Banach algebra, inside this cochain complex there is a subcomplex of entire cochains satisfying the following growth condition

$$
\sum_{n \geq 0}\left\|f_{2 n}\right\| n ! r^{n}<\infty
$$

for all positive real $r$. There is an identical condition to be satisfied by odd cochains. The cohomology of this complex is the entire cyclic cohomology. We can define a similar theory when we consider the same growth condition but we require that the cochains be simplicially normalized. In other words, if $f$ is a cochain, we require that

$$
f_{n}\left(a_{0}, a_{1}, \ldots, a_{n}\right)=0
$$

whenever $a_{i}=1$ for some $1 \leq i \leq n$. It is a standard statement of the cyclic cohomology that replacing cochains by normalized cochains is a quasi-isomorphism [LQ], and in fact the same is true in theory of simplicial complexes [MacL]. It is our aim in this paper to present a proof of a similar statement in the case of entire cyclic cohomology.

In our approach we use the fact that we can associate with a unital algebra $A$ a universal algebra $Q A=A * A$, the Cuntz algebra, which is defined as the free product of $A$ by itself. Then there is a universal model for the simplicial normalization problem, which may be described as follows. On the one hand we may consider the free product with amalgamation over the identity element of $A$, thus obtaining the Cuntz algebra $Q A$ in the category of unital algebras. On the other hand, following Connes, we can adjoin the unit to $A$, thus obtaining the augmented algebra $\tilde{A}$, and then we can form the Cuntz algebra $Q \tilde{A}$ where the amalgamation is taken over the new unit. We show that, roughly speaking, the two ways of associating the Cuntz algebra with $A$ are equivalent from the point of view of entire cyclic cohomology. More precisely, we show that there is a homotopy between $Q \tilde{A}$ and $\widetilde{Q A}=\mathbf{C} \oplus Q A$ which induces a quasi-isomorphism on the entire cyclic cohomology. We do this by constructing a family $\phi_{t}$ of homomorphisms of $Q \tilde{A}$ and studying the effect of its action on the supertraces on a completion $Q_{\epsilon} \tilde{A}$ of $Q \tilde{A}$. We show that this family of homomorphisms respects the homotopy class of a supertrace. Since we are interested in the case where $A$ is a Banach algebra, we need to introduce a suitable topology on the Cuntz algebra and consider homomorphisms and supertraces which are continuous with respect to that topology.

The reason for introducing supertraces on the Cuntz algebra is that they lead to certain entire cyclic cocycles and, moreover, in each entire cyclic cohomology class there is an entire cyclic cochain coming from a supertrace on $Q A$. Even more, supertraces that are homotopic in a certain sense correspond to entire cyclic cocycles which are in the same entire cyclic cohomology class. One of the difficulties which we have to face is the problem of adapting the purely algebraic formalism of CuntzQuillen to the present topological situation. 
Even if the problem that we address in this paper may seem technical, the method which we employ should prove useful in other situations. We see the proof of simplicial normalization property of entire cyclic cohomology as a first step towards adapting the Cuntz-Quillen formalism to the study of cohomology theories of topological algebras. As another example of a possible application of this method we present a proof of the homotopy invariance of the entire cyclic cohomology.

\section{Universal algebras associated with an algebra}

We begin with a discussion of two universal algebras associated with an algebra $A$, namely the algebra of non-commutative differential forms $\Omega A$ and the Cuntz algebra $Q A$. In our exposition we use the approach presented in the series of papers by Cuntz and Quillen [CuQ1, CuQ2, CuQ3], to which we refer the reader for more details.

Let $A$ be a unital algebra. We shall denote by $\Omega^{1} A$ the $A$-bimodule of 1 forms over $A$. Denoting by $d$ the canonical derivation of $A$ with values in $\Omega^{1} A$, we have that $\Omega^{1} A$ is spanned by the symbols $a_{0} d a_{1}$, for $a_{0}$ and $a_{1}$ in $A$. The space of $n$-forms is then defined by

$$
\Omega^{n} A=\Omega^{1} A \otimes_{A} \cdots \otimes_{A} \Omega^{1} A
$$

Let $\Omega A=\oplus_{n} \Omega^{n} A$. We define a product on this graded space as follows. Let $\omega_{1} \cdots \omega_{n}$, for $\omega_{i} \in \Omega^{1} A$, be the image of $\omega_{1} \otimes \cdots \otimes \omega_{n}$ in $\Omega_{n} A$. Then put

$$
\left(\omega_{1} \cdots \omega_{n}\right)\left(\eta_{1} \cdots \eta_{m}\right)=\omega_{1} \cdots \eta_{m}
$$

We extend the differential $d$ to $\Omega A$ so that it becomes a differential graded algebra with respect to the above product.

An equivalent description of the space of forms is provided by a family of isomorphisms

$$
\Omega^{n} A=A \otimes \bar{A}^{\otimes n}
$$

where $\bar{A}=A / \mathbf{C}$.

The Hochschild boundary operator $b$ is defined on homogeneous forms of positive degree by

$$
b(\omega d a)=(-1)^{|\omega|}(\omega a-a \omega)=(-1)^{|\omega|}[\omega, a]
$$

In degree zero we put $b=0$. Thus $b$ is a differential of degree -1 and square zero. The Karoubi operator $\kappa$ is defined by

$$
\kappa(\omega d a)=(-1)^{|\omega|} d a \omega
$$

This operator of degree zero satisfies the following 'homotopy' formula

$$
b d+d b=1-\kappa
$$

Moreover, $\kappa$ commutes with both differentials $b$ and $d$. Finally, on the space of $n$-forms $\Omega^{n} A$, the operator $B$ of Connes is defined by

$$
B=\sum_{i=0}^{n} \kappa^{i} d
$$


The operator $B$ is a degree +1 differential, i.e. $B^{2}=0$. Equipped with the two differentials $b$ and $B$ the DG algebra of forms $\Omega A$ becomes a mixed complex in the sense of Kassel [Ka1].

The homotopy formula (2.3) suggests that we can treat the operator $1-\kappa$ as the formal analogue of the Laplacian on a Riemannian manifold. In particular, we have direct sum decomposition of the algebra of forms into the eigenspaces of the operator $\kappa$. Of most interest is the (generalized) eigenspace in $\Omega A$ where $\kappa$ has eigenvalue 1 . Denoting by $P$ the spectral projection onto this eigenspace we may write

$$
\Omega A=P \Omega A \oplus P^{\perp} \Omega A
$$

Using a formal analogy with the situation of a Riemannian manifold, the subcomplex $P \Omega A$ is called the space of harmonic forms. Moreover, the complexes $\Omega A$ and $P \Omega A$ are quasi-isomorphic from the point of view of cyclic homotopy types. The relevance of this decomposition in the entire cyclic cohomology will be explained in the following sections.

We may consider the following natural algebraic completion of the algebra $\Omega A$. The subspace $J=\bigoplus_{n \geq 1} \Omega^{n} A$ of forms of degree at least 1 is an ideal in $\Omega A$ which gives a decreasing filtration of $\Omega A$

$$
\ldots J^{n} \subset \ldots \subset J^{2} \subset J \subset \Omega A
$$

and we let $\hat{\Omega} A$ be the $J$-adic completion of $\Omega A$

$$
\hat{\Omega} A=\lim _{\longleftarrow} \Omega A / J^{n}
$$

It is not difficult to see that $\hat{\Omega} A=\prod_{n \geq 0} \Omega^{n} A$. We shall make use of this algebraic completion when describing various other topologies that can be introduced on the algebra $\Omega A$.

If the algebra $A$ is nonunital we let $\tilde{A}$ be the augmented algebra $\tilde{A}=\mathbf{C} \oplus A$. We then form the DG algebra of non commutative differential forms $\Omega \tilde{A}$ as above, but we note that in the present case the algebra of forms has the following new properties. First, this algebra is itself augmented, i.e.

$$
\Omega \tilde{A}=\mathbf{C} \oplus \bar{\Omega} \tilde{A}
$$

where the reduced part $\bar{\Omega} \tilde{A}$ is the canonical nonunital differential graded algebra generated by $A$. Furthermore, there is an isomorphism $A^{\otimes n+1} \oplus A^{\otimes n} \rightarrow \bar{\Omega}^{n} \tilde{A}$. Thus all the standard operators on $\bar{\Omega} \tilde{A}$ may be given by suitable $2 \times 2$ matrices. In particular we have that

$$
\begin{array}{cc}
\tilde{d}=\left(\begin{array}{cc}
0 & 0 \\
1 & 0
\end{array}\right), & \tilde{b}=\left(\begin{array}{cc}
b & 1-\lambda \\
0 & -b^{\prime}
\end{array}\right), \\
\tilde{\kappa}=\left(\begin{array}{cc}
\lambda & 0 \\
b^{\prime}-b & \lambda
\end{array}\right), & \tilde{B}=\left(\begin{array}{cc}
0 & 0 \\
N_{\lambda} & 0
\end{array}\right),
\end{array}
$$


where

$$
\begin{aligned}
b^{\prime}\left(a_{0}, \ldots, a_{n}\right) & =\sum_{i=0}^{n-1}(-1)^{i}\left(a_{0}, \ldots, a_{i} a_{i+1}, \ldots, a_{n}\right) \\
\lambda\left(a_{0}, \ldots, a_{n}\right) & =(-1)^{n}\left(a_{n}, a_{0}, \ldots, a_{n-1}\right)
\end{aligned}
$$

and $N_{\lambda}=\sum_{i=1}^{n-1} \lambda^{i}$ in degree $n$. The algebra $\Omega \tilde{A}$ equipped with the operators described above is identical to the first two columns in the classical double complex of Connes-Loday-Quillen-Tsygan.

We now pass to the description of the Cuntz algebra $Q A$ associated with an algebra $A$. If $A$ is a unital algebra then the Cuntz algebra $Q A$ is defined as the free product $Q A=A * A$ in the category of unital algebras, i.e., with amalgamation over the identity of $A$. There are two canonical homomorphisms $\iota, \iota^{\gamma}$ from $A$ to $Q A$ and a canonical automorphism of $Q A$ of order two: $\omega \mapsto \omega^{\gamma}$ which interchanges $\iota$ and $\iota^{\gamma}$. The algebra $Q A$ is a superalgebra which means that $Q A$ is $\mathbf{Z} / 2$-graded and the grading is compatible with multiplication.

For $a \in A$, let $p(a)$ and $q(a)$ denote the even and odd components of $\iota(a)$ with respect to the $\mathbf{Z} / 2$-grading of $Q A$. If we put $a=\iota(a)$ and $a^{\gamma}=\iota^{\gamma}(a)$ then $p(a)=\left(a+a^{\gamma}\right) / 2$ and $q(a)=\left(a-a^{\gamma}\right) / 2$. The following relations determine the product in $Q A$.

$$
\begin{aligned}
p\left(a_{1} a_{2}\right) & =p\left(a_{1}\right) p\left(a_{2}\right)+q\left(a_{1}\right) q\left(a_{2}\right) \\
q\left(a_{1} a_{2}\right) & =p\left(a_{1}\right) q\left(a_{2}\right)+q\left(a_{1}\right) p\left(a_{2}\right)
\end{aligned}
$$

We deduce from these relations that any element in $Q A$ may be represented as a linear combination of elements of the form $p\left(a_{0}\right) q\left(a_{1}\right) \cdots q\left(a_{n}\right)$. Note finally that the unit of the algebra $A$ is also the unit of $Q A$ and we have $p(1)=1, q(1)=0$.

There is a canonical map $Q A \rightarrow A$ which identifies the two copies of the algebra $A$ inside $Q A$. This map is called the folding map, and its kernel is denoted by $q A$. As in the case of the algebra of forms, there is a natural completion of this algebra which is the inverse limit of the system $\hat{Q} A=\lim Q A / q A^{n}$. This completion is again a $\mathbf{Z} / 2$-graded algebra.

There is a linear isomorphism $\mu$ between the underlying graded vector space of the Cuntz algebra $Q A$ and the graded vector space of forms $\Omega A$ which on generators is defined by

$$
\mu: p\left(a_{0}\right) q\left(a_{1}\right) \cdots q\left(a_{n}\right) \mapsto a_{0} d a_{1} \cdots d a_{n}
$$

In particular, if $a \in A$ as a subalgebra of $Q A$ then $\mu(a)=\mu(p(a))+\mu(q(a))=$ $a+d a \in \Omega A$.

The map $\mu$ becomes an isomorphism of $\mathbf{Z} / 2$-graded algebras when the algebra $\Omega A$ is equipped with the Fedosov product defined as follows. If $\omega$ and $\eta$ are two homogeneous elements of $\Omega A$ we put

$$
\omega \circ \eta=\omega \eta-(-1)^{|\omega|} d \omega d \eta
$$

We may therefore think of the Cuntz algebra as a deformation of the algebra of differential forms $\Omega A$ equipped with the Fedosov product. In particular, under this 
isomorphism, the ideal $J$ in $\Omega A$ is identified with the folding ideal $q A$ of the Cuntz algebra.

Using this isomorphism it is easy to see that if $\tilde{A}$ is an augmented algebra then $Q \tilde{A}$ is also augmented

$$
Q \tilde{A}=\mathbf{C} \oplus \bar{Q} \tilde{A}
$$

where $\bar{Q} \tilde{A}$, the reduced part of the Cuntz algebra, is the Cuntz algebra associated with $A$ in the nonunital category.

If $A$ is in fact a unital algebra then the unit 1 of $A$ has two images $u$ and $u^{\gamma}$ in $Q \tilde{A}$ which are idempotents. The corresponding involutions $f=2 u-1$ and $f^{\gamma}$ generate inside the algebra $\hat{Q} \tilde{A}$ an algebra which is isomorphic to $\hat{Q} \tilde{\mathbf{C}}$, the group algebra of the infinite dihedral group. We shall record its basic properties for future use $[\mathrm{Cu} 2]$.

The dihedral group generated by the two involutions $f$ and $f^{\gamma}$ may be presented using the following two generators. First, let

$$
L=\log \left(f f^{\gamma}\right)=-\sum_{n \geq 1}(2 f q(f))^{n} / n
$$

Since $\left(f f^{\gamma}\right)^{\gamma}=f^{\gamma} f=\left(f f^{\gamma}\right)^{-1}$, we have on the one hand $\left(e^{L}\right)^{\gamma}=e^{-L}$ while on the other $\left(e^{L}\right)^{\gamma}=e^{\left(L^{\gamma}\right)}$ from which follows that $L^{\gamma}=-L$, i.e. $L$ is odd with respect to the canonical automorphism $\gamma$.

We put $W=e^{L / 2}$. Since $f f^{\gamma}=\exp L$ we have

$$
W f^{\gamma}=W f e^{L}=e^{L / 2} e^{-L} f=e^{-L / 2} f=f e^{L / 2}
$$

so that

$$
f^{\gamma}=W^{-1} f W
$$

It is now a good moment to state explicit formulae for $L$ and $W$ that we will need later $[\mathrm{B}]$.

2.6 Proposition. Let $L=\log \left(f f^{\gamma}\right)=-\sum_{n \geq 1}(2 f q(f))^{n} / n$. Then

$$
\log \left(f f^{\gamma}\right)=-\sum_{i \geq 1} \frac{((i-1) !)^{2}}{(2 i-1) !} p(f) q(f)^{2 i-1} .
$$

Let us now define $W_{t}=\exp (t L / 2)$ for $t \in[0,1]$.

2.7 Proposition. For all $t \in[0,1]$ we have

$$
W_{t}=1+\sum_{n \geq 1}(-1)^{n} 2^{2 n-1}\left(\begin{array}{c}
t / 2+n-1 \\
2 n-1
\end{array}\right)\left((t / 2 n) q(f)^{2 n}+p(f) q(f)^{2 n-1}\right)
$$

Moreover, we have that $W_{-t}=W_{t}^{\gamma}$. Thus $p\left(W_{-t}\right)=p\left(W_{t}\right)$ and $q\left(W_{-t}\right)=-q\left(W_{t}\right)$. 
Proof. The first part of the proposition follows if we use the binomial series expansion for $W_{t}=e^{t L}=\left(f f^{\gamma}\right)^{t / 2}=(1-2 f q)^{t / 2}$, together with combinatorial formulae in [B].

We have seen that $L^{\gamma}=-L$, from which it follows that

$$
W_{t}^{\gamma}=\left(e^{t L / 2}\right)^{\gamma}=e^{t L^{\gamma} / 2}=e^{-t L / 2}=W_{-t}
$$

We note that when we compare this statement with the explicit formula for $W_{t}$ given above, we find the following symmetry of the binomial coefficients which appear in the decomposition of $W_{t}$

$$
\left(\begin{array}{c}
-t / 2+n-1 \\
2 n-1
\end{array}\right)=(-1)^{2 n-1}\left(\begin{array}{c}
t / 2+n-1 \\
2 n-1
\end{array}\right)
$$

which is not very difficult to check directly. The second statement of the present Proposition is now clear.

\section{Topology on $\Omega A$ and $Q A$}

Let $A$ be a unital Banach algebra equipped with a norm \|\| . Let us denote by $\bar{A}=$ $A / \mathbf{C}$ the quotient space which is a Banach space equipped with the quotient space norm. The space of one-forms $\Omega^{1} A$ becomes a Banach space with the completed projective tensor product norm $\Omega^{1} A=A \hat{\otimes}_{\pi} \bar{A}$. The operator $d: A \rightarrow \Omega^{1} A$ is now a continuous operator of unit norm. For any $n \geq 1$ we equip the space of $n$-forms $\Omega^{n} A$ with the topology of the completed projective tensor product [Arv, p. $260 \mathrm{ff}$ ]. The differential $d$ becomes a continuous derivation of unit norm and the product (2.2) is now a continuous map.

Given this topological structure, we may now equip the differential graded algebra $\Omega A=\bigoplus_{n \geq 0} \Omega^{n} A$ with the following family of norms \|\|$_{r}$ parametrized by a positive real $r$. If $\omega=\sum_{n \geq 0} \omega_{n}$ we put

$$
\|\omega\|_{r}=\sum_{n \geq 0}\left\|\omega_{n}\right\|_{\pi} r^{n}
$$

The derivation $d$ becomes a continuous map $d:\left(\Omega A,\|\|_{r}\right) \rightarrow\left(\Omega A,\|\|_{r}\right)$. In particular, $\|d a\|_{r} \leq r\|a\|$ for $a \in A$. If $\omega_{n}$ is a homogeneous form of degree $n$ then

$$
\left\|\omega_{n}\right\|_{r}=r^{n}\left\|\omega_{n}\right\|, \quad\left\|d \omega_{n}\right\|_{r}=r^{n+1}\left\|d \omega_{n}\right\| \leq r^{n+1}\left\|\omega_{n}\right\|=r\|\omega\|_{r}
$$

For any two $r^{\prime}<r$, let

$$
f_{r r^{\prime}}:\left(\Omega A,\|\|_{r}\right) \longrightarrow\left(\Omega A,\|\|_{r^{\prime}}\right)
$$

be the identity map, which in this case is norm decreasing. This way we obtain a direct system $\left\{\left(\Omega A,\|\|_{r}\right), f_{r r^{\prime}}\right\}$. Let $\Omega_{r} A$ denote the completion of the space 
$\left(\Omega A\|\|_{r}\right)$ with respect to the norm \|\|$_{r}$. This is now a Banach algebra, which means that for any two forms $\omega, \eta$ in $\Omega_{r} A$ we have [Arv, p. 262]

$$
\|\omega \eta\|_{r} \leq\|\omega\|_{r}\|\eta\|_{r}
$$

This formula follows directly from the definitions of the product (2.2) and the norm \|\|$_{r}$.

There is an induced direct system $\left(\Omega_{r} A, f_{r r^{\prime}}\right)$ of Banach algebras. We denote by $\Omega_{\epsilon} A$ the direct limit of this system as $r \rightarrow 0$ :

$$
\Omega_{\epsilon} A=\lim _{\longrightarrow} \Omega_{r} A
$$

Finally, we denote by $f_{r}$ the canonical maps $f_{r}: \Omega_{r} A \rightarrow \Omega_{\epsilon} A$, which are continuous algebra homomorphisms. The derivation $d$ extends to a continuous derivation $d_{r}$ : $\Omega_{r} A \longrightarrow \Omega_{r} A$. The composite $f_{r} \circ d_{r}: \Omega_{r} A \longrightarrow \Omega_{\epsilon} A$ is a continuous derivation of $\Omega_{r} A$ with values in $\Omega_{\epsilon} A$ and all derivations $f_{r} \circ d_{r}$ are compatible with the maps $f_{r^{\prime} r}$. Thus this family of derivations is equivalent to a continuous derivation $d: \Omega_{\epsilon} A \longrightarrow \Omega_{\epsilon} A$ which makes $\Omega_{\epsilon} A$ into a topological differential graded algebra equipped with the locally convex topology induced by the topologies on $\Omega_{r} A$.

In the case when $A$ is a unital Banach algebra, for any positive real $r$, the completion $\Omega_{r} \tilde{A}$ is an augmented Banach algebra

$$
\Omega_{r} \tilde{A}=\mathbf{C} \oplus \bar{Q}_{r} \tilde{A}
$$

from which it follows that also the direct limit of the corresponding direct system is an augmented algebra

$$
\Omega_{\epsilon} \tilde{A}=\mathbf{C} \oplus \bar{\Omega}_{\epsilon} \tilde{A}
$$

We now define a topology on the Cuntz algebra $Q A$ using the families of norms \|\|$_{r}$ on $\Omega A$ together with the isomorphism $\mu$ between the two superalgebras. And so if $\omega$ is an element of the Cuntz algebra $Q A$ then its norm $\|\omega\|_{Q_{r}}$, for any positive $r$ is defined by

$$
\|\omega\|_{Q_{r}}=\|\mu(\omega)\|_{r}
$$

In particular, for $a \in A,\|a\|_{Q_{r}}=\|a+d a\|_{r}=\|a\|+r\|d a\|$. This means that the isomorphism $\mu$ induces a new norm on the the algebra $A$ which is equivalent to the original Banach norm on $A$.

We shall need the following estimates for the Fedosov product.

3.1 Lemma. For any $r>0$, and for any $\omega, \eta \in \Omega_{r} A$ we have

$$
\|\omega \circ \eta\|_{r} \leq C_{r}\|\omega\|_{r}\|\eta\|_{r}
$$

where $C_{r}=1+r^{2}$ and $\circ$ denotes the Fedosov product of forms. If either of the forms $\omega, \eta$ is closed then this estimate reduces to

$$
\|\omega \circ \eta\|_{r} \leq\|\omega\|_{r}\|\eta\|_{r}
$$


Proof. If $\omega=\sum \omega_{n}$ and $\eta=\sum \eta_{n}$ then

$$
\begin{aligned}
\omega \circ \eta & =\sum_{n \geq 0} \sum_{i+j=n} \omega_{i} \circ \eta_{j} \\
& =\sum_{n \geq 0} \sum_{i+j=n} \omega_{i} \eta_{j}-(-1)^{\left|\omega_{i}\right|} d \omega_{i} d \eta_{j}
\end{aligned}
$$

The norm \|\|$_{Q_{r}}$ of this form may then be estimated as follows.

$$
\begin{aligned}
\|\omega \circ \eta\|_{r} & \leq \sum_{n \geq 0}\left(\sum_{i+j=n}\left\|\omega_{i} \eta_{j}\right\|\right) r^{n}+\sum_{n \geq 0}\left(\sum_{i+j=n}\left\|d \omega_{i} d \eta_{j}\right\|\right) r^{n+2} \\
& \leq \sum_{n \geq 0}\left(\sum_{i+j=n}\left\|\omega_{i}\right\|\left\|\eta_{j}\right\|_{\pi}\right) r^{n}+r^{2} \sum_{n \geq 0}\left(\sum_{i+j=n}\left\|\omega_{i}\right\|\left\|\eta_{j}\right\|\right) r^{n} \\
& =\left(1+r^{2}\right)\|\omega\|_{r}\|\eta\|_{r}
\end{aligned}
$$

The last statement of the lemma is now clear.

3.2 Corollary. If $x, y \in Q_{r} A$ then

$$
\|x y\|_{Q_{r}}=\|\mu(x) \circ \mu(y)\|_{r} \leq\left(1+r^{2}\right)\|\mu(x)\|\left\|_{r}\right\| \mu(y)\left\|_{r}=C_{r}\right\| x\left\|\left.\right|_{Q_{r}}\right\| y \|_{Q_{r}}
$$

This means in particular that multiplication in the Cuntz algebra is continuous in this topology.

We now introduce a direct system of normed spaces $\left(Q A,\|\|_{Q_{r}}\right)$ as in the case of the algebra of differential forms. For any pair $r^{\prime}<r$ there is a map

$$
f_{r r^{\prime}}:\left(Q A,\|\|_{Q_{r}}\right) \longrightarrow\left(Q A,\|\|_{Q_{r^{\prime}}}\right)
$$

which is the identity map. Using the estimates

$$
\|\omega\|_{Q_{r^{\prime}}}=\|\mu(\omega)\|_{r^{\prime}}<\|\mu(\omega)\|_{r}=\|\omega\|_{Q_{r}}
$$

we see that for any two $r^{\prime}<r$ the map $f_{r r^{\prime}}$ is continuous and norm decreasing. Let us denote by $Q_{r} A$ the completion of the space $\left(Q A,\|\|_{Q_{r}}\right)$, then there is a direct system $\left(Q_{r} A, f_{r r^{\prime}}\right)$ of complete normed spaces. We denote by $Q_{\epsilon} A$ its direct limit taken as $r \rightarrow 0$.

As before, in the case of a unital Banach algebra $A$, the Cuntz algebra $Q_{\epsilon} \tilde{A}$ is also an augmented algebra. This follows from the fact that for any $r$ we have

$$
Q_{r} \tilde{A}=\mathbf{C} \oplus \bar{Q}_{r} \tilde{A}
$$


so that we have

$$
Q_{\epsilon} \tilde{A}=\mathbf{C} \oplus \bar{Q}_{\epsilon} \tilde{A}
$$

where $\bar{Q}_{\epsilon} \tilde{A}$ is the direct limit of the direct subsystem $\bar{Q}_{r} \tilde{A}$.

Our last task in this section is to show that the elements $L$ and $W_{t}$ of the algebraic completion $\hat{Q} \tilde{A}$ are in fact elements of the locally convex algebra $Q_{\epsilon} \tilde{A}$.

3.3 Proposition. If respecting the notations introduces previously we define

$$
L=\log f f^{\gamma}=-\sum_{n \geq 1} \frac{1}{n}(2 f q(f))^{n}
$$

and $W_{t}=\exp t L / 2, t \in[0,1]$, then $L$ and $W_{t}$ are elements of $Q_{\epsilon} \tilde{A}$. More precisely, they belong to $Q_{r} \tilde{A}$ for all $r<1 /\|q(f)\|$.

Proof. Recall from lemma 2.6 that $L$ is given by the following explicit formula

$$
\log \left(f f^{\gamma}\right)=-\sum_{n \geq 1} \frac{((n-1) !)^{2}}{(2 n-1) !} p(f) q(f)^{2 n-1}
$$

Since

$$
\frac{((n-1) !)^{2}}{(2 n-1) !}=\frac{(n-1) !}{n(n+1) \ldots(2 n-1)} \leq 1
$$

for any $n>1$, we have that

$$
\begin{aligned}
\|L\|_{Q_{r}} & \leq \sum_{n \geq 1}\left\|p(f) q(f)^{2 n-1}\right\|_{Q_{r}} \\
& \leq \sum_{n \geq 1}\|p(f) q(f)\|^{2 n-1} r^{2 n-1} .
\end{aligned}
$$

The last series above is clearly convergent for $r<1 /\|q(f)\|$, which proves the first part of our statement.

We note here for future use that the same estimate may be written as

$$
\|L\|_{Q_{r}} \leq r\|p(f) q(f)\| \sum_{n \geq 0}\|q(f)\|^{2 n} r^{2 n}=\frac{r\|p(f) q(f)\|}{1-(\|q(f)\| r)^{2}}
$$

Thus if we restrict $r$ to $r<r_{0}=1 / 2\|q(f)\|$, say, we may write

$$
\|L\|_{Q_{r}} \leq r N
$$

where $N=(4 / 3)\|p(f) q(f)\|$.

We now turn to the family of operators $W_{t}=\exp (t L / 2)$. Note that if $\omega \in Q_{r} \tilde{A}$ then also $e^{\omega} \in Q_{r} \tilde{A}$. Indeed, we have

$$
\left\|e^{\omega}\right\|_{Q_{r}} \leq \sum_{n \geq 0} C_{r}^{n-1} \frac{\|\omega\|_{Q_{r}}^{n}}{n !} \leq \exp \left(C_{r}\|\omega\|_{Q_{r}}\right)<\infty
$$


since $\|\omega\|_{Q_{r}}$ is finite by hypothesis. This is sufficient to prove the second part of our hypothesis.

It is now a good moment to record a more precise estimate of the norm of the odd part of $W_{t}$ which will be needed in what follows.

3.5 Proposition. For any $t \in[0,1]$ and any $r<r_{0}=1 / 2\|q\|$ we have

$$
\left\|q\left(W_{t}\right)\right\|_{Q_{r}} \leq r N
$$

where the constant $N$ is as in the formula (3.4).

Proof. From our explicit expression for $W_{t}$ it follows that

$$
q\left(W_{t}\right)=\sum_{n \geq 1} c_{n} p(f) q(f)^{2 n-1}
$$

where

$$
c_{n}=(-1)^{n} 2^{2 n-1}\left(\begin{array}{c}
t / 2+n-1 \\
2 n-1
\end{array}\right)
$$

The binomial coefficient above may be estimated as follows. Note first that

$$
\frac{\left|c_{n}\right|}{\left|c_{n-1}\right|}=\frac{|4(t / 2+n-1)(t / 2-n+1)|}{(2 n-2)(2 n-1)}=\frac{4\left|(n-1)^{2}-(t / 2)^{2}\right|}{2(n-1)(2 n-1)} \leq 1
$$

so that $\left|c_{n}\right| \leq\left|c_{n-1}\right|$ for all $n \geq 1$. Since $\left|c_{1}\right| \leq 1$ we have that $\left|c_{n}\right| \leq 1$ for all $n$.

It now follows that

$$
\left\|q\left(W_{t}\right)\right\|_{Q_{r}} \leq \sum_{n \geq 1}\left\|c_{n} p(f) q(f)^{2 n-1}\right\|_{Q_{r}} \leq r\|p(f) q(f)\| \sum_{n \geq 0}\|q(f)\|^{2 n} r^{2 n} \leq r N
$$

for $r \leq 1 / 2\|q(f)\|$.

\section{A homotopy}

A first step in the proof of the simplicial normalization theorem for entire cyclic cohomology will be to show that the algebras $Q_{\epsilon} \tilde{A}$ and $\mathbf{C} \oplus Q_{\epsilon} A$ are homotopy equivalent. At the same time we shall establish a homotopy equivalence between the superalgebras $\bar{Q}_{\epsilon} \tilde{A}$ and $Q_{\epsilon} A$. We establish the required homotopy using a certain family $\left\{\phi_{t}\right\}$ of continuous superalgebra homomorphisms $\phi_{t}: Q_{\epsilon} \tilde{A} \rightarrow Q_{\epsilon} \tilde{A}$. The definition and properties of the family $\phi_{t}$, which have been suggested to us by J. Cuntz (see also [Cu2]), will depend in an important way on the properties of the operators $L$ and $W_{t}$ established in the previous section.

For any $t \in[0,1]$ we put

$$
\begin{aligned}
a & \mapsto a_{t}=e^{-t L / 2} a e^{t L / 2}=W_{-t} a W_{t} \\
a^{\gamma} & \mapsto a_{t}^{\gamma}=e^{t L / 2} a^{\gamma} e^{-t L / 2}=W_{t} a^{\gamma} W_{-t}
\end{aligned}
$$


We note that the first of the two maps is a homomorphism from the algebra $A$ to the underlying algebra of $Q_{\epsilon} \tilde{A}$ and that the other map is induced from the first by applying the canonical automorphism $\gamma$ which sends $a_{t}$ to $a_{t}^{\gamma}$. This pair of algebra homomorphisms extends to the following family of homomorphisms $\phi_{t}: Q \widetilde{A} \longrightarrow$ $Q_{\epsilon} \tilde{A}$. For $\omega \in Q_{\epsilon} \tilde{A}, \omega=p\left(a_{0}\right) q\left(a_{1}\right) \cdots q\left(a_{n}\right)$, we have

$$
\phi_{t}(\omega)=\omega_{t}=p_{t}\left(a_{0}\right) q_{t}\left(a_{1}\right) \cdots q_{t}\left(a_{n}\right)
$$

where

$$
\begin{aligned}
p_{t}(a) & =p\left(a_{t}\right)=1 / 2\left(a_{t}+a_{t}^{\gamma}\right) \\
q_{t}(a) & =q\left(a_{t}\right)=1 / 2\left(a_{t}-a_{t}^{\gamma}\right)
\end{aligned}
$$

We remark that $\phi_{0}$ is the identity map whereas $\phi_{1}$ is the map which sends $f$ to $f^{\gamma}$ by $(2.5)$.

4.2 TheOrem. The family $\phi_{t}$ defined above is a family of continuous superalgebra homomorphisms from $Q_{\epsilon} \tilde{A}$ to $Q_{\epsilon} \tilde{A}$.

The proof depends on a few estimates, which we derive below. Before we begin we note that, for any $t \in[0,1]$ and any $\lambda \in \mathbf{C}, p_{t}(\lambda)=\lambda$ and $q_{t}(\lambda)=0$ so that $\phi_{t}$ acts nontrivially only on the reduced part $\bar{Q}_{\epsilon} \tilde{A}$ of the algebra $Q_{\epsilon} \tilde{A}$, and therefore we shall concentrate on this case.

First we need to estimate norms \|\|$_{Q_{r}}$ of $p_{t}(a)$ and $q_{t}(a)$, for any $a \in A \subset \tilde{A}$.

4.3 Proposition. For any $t \in[0,1]$ and any $r<r_{0}=1 / 2\|q(f)\|$ we have the following estimates for any $a \in A$

$$
\begin{aligned}
& \left\|p\left(a_{t}\right)\right\|_{Q_{r}} \leq M^{\prime}\|p(a)\|_{Q_{r}} \\
& \left\|q\left(a_{t}\right)\right\|_{Q_{r}} \leq M^{\prime \prime}\|q(a)\| \|_{Q_{r}}
\end{aligned}
$$

where $M^{\prime}$ and $M^{\prime \prime}$ are constants which only depend on $r_{0}$. We may then replace them with $M=\max \left(M^{\prime}, M^{\prime \prime}\right)$.

Proof. As both estimates are proved in the same way, we give the details of the proof of the second of the two.

Using the definition of the product in the Cuntz algebra we may write that the odd part $q\left(a_{t}\right)$ of $a_{t}$ is given by

$$
\begin{aligned}
q\left(a_{t}\right)= & p\left(W_{t}\right) p(a) q\left(W_{t}\right)+p\left(W_{t}\right) q(a) p\left(W_{t}\right) \\
& -q\left(W_{t}\right) p(a) p\left(W_{t}\right)-q\left(W_{t}\right) q(a) q\left(W_{t}\right)
\end{aligned}
$$

where we have used the fact that $p\left(W_{-t}\right)=p\left(W_{t}\right)$ and $q\left(W_{-t}\right)=-q\left(W_{t}\right)$. The terms containing $q(a)$ present no problem from the point of view of our estimates, so let us concentrate on the other two terms. Using proposition 3.5 we have that

$$
\begin{aligned}
\left\|p\left(W_{t}\right) p(a) q\left(W_{t}\right)\right\|_{Q_{r}} & \leq C_{r}^{2}\left\|p\left(W_{t}\right)\right\|_{Q_{r}} N r\|p(a)\|_{Q_{r}} \\
& =C_{r}^{2}\left\|p\left(W_{t}\right)\right\|_{Q_{r}} N\|q(a)\|_{Q_{r}} \\
& =M_{1}\|q(a)\|_{Q_{r}}
\end{aligned}
$$


for any $r<r_{0}$ and any $a \in A$, and similarly with the second term containing $p(a)$. We have used here that $r\|p(a)\|_{Q_{r}}=\|q(a)\|_{Q_{r}}$ for $a \in A$. Thus the norm of $q\left(a_{t}\right)$ is bounded from above by the sum of four terms of the form $M_{i}\|q(a)\|_{Q_{r}}$, which leads to the required formula when we put $M^{\prime \prime}$ to be the sum of the $M_{i}$ 's, for $i=1, \ldots, 4$.

It follows that if $\omega_{n}=p\left(a_{0}\right) q\left(a_{1}\right) \ldots q\left(a_{n}\right)$ then

$$
\left\|\omega_{t, n}\right\|_{Q_{r}} \leq M^{n+1} r^{n}\left\|p\left(a_{0}\right)\right\|\left\|q\left(a_{1}\right)\right\| \cdots\left\|q\left(a_{n}\right)\right\|=M^{n+1} r^{n}\left\|\omega_{n}\right\|
$$

Let us now assume that $\omega \in \bar{Q}_{\epsilon} \tilde{A}$. This means that there exists an $r$, which may always be chosen to be smaller than $r_{0}$, such that $\omega \in \bar{Q}_{r} \tilde{A}$, i.e. that $\omega=\sum_{n \geq 0} \omega_{n}$ and

$$
\begin{aligned}
\|\omega\|_{Q_{r}} & =\sum_{n \geq 0}\left\|\omega_{n}\right\|_{Q_{r}} \\
& =\sum_{n \geq 0}\left\|\omega_{n}\right\| r^{n}<\infty
\end{aligned}
$$

We now want to find an estimate of the norm of $\omega_{t}$. We have

$$
\begin{aligned}
\left\|\omega_{t}\right\|_{Q_{r^{\prime}}} & =\left\|\sum_{n \geq 0} \omega_{t, n}\right\|_{Q_{r^{\prime}}} \leq \sum_{n \geq 0} M^{n+1}\left\|\omega_{n}\right\|_{Q_{r^{\prime}}} \\
& =M \sum_{n \geq 0}\left\|\omega_{n}\right\| M^{n} r^{\prime n} \leq M\|\omega\|_{Q_{r}}
\end{aligned}
$$

if $r^{\prime} \leq r / M$. We have thus proved that for any $\omega \in \bar{Q}_{\epsilon} \tilde{A}$ there exist two norms \|\|$_{Q_{r^{\prime}}}$ and \|\|$_{Q_{r}}$, where $r^{\prime}<r<r_{0}$, such that

$$
\left\|\phi_{t}(\omega)\right\|_{Q_{r^{\prime}}} \leq M\|\omega\|_{Q_{r}}
$$

where $M$ is the constant determined in the estimates of the norms of $p_{t}(a)$ and $q_{t}(a)$ above. But this means that, for any $t \in[0,1], \phi_{t}$ is a continuous homomorphism $Q_{\epsilon} \tilde{A} \rightarrow Q_{\epsilon} \tilde{A}$ in the locally convex topology with which $Q_{\epsilon} \tilde{A}$ is equipped as the direct limit of the system $\left(Q_{r} \tilde{\tilde{A}}, f_{r r^{\prime}}\right)$. This gives the proof of Theorem 4.2 .

It is clear that the folding map in $Q \tilde{\mathbf{C}}$

$$
0 \rightarrow q \tilde{\mathbf{C}} \rightarrow Q \tilde{\mathbf{C}} \rightarrow \tilde{\mathbf{C}} \rightarrow 0
$$

induces a canonical projection

$$
Q \tilde{A} \longrightarrow \mathbf{C} \oplus Q A
$$

The kernel of this projection is an ideal $I$ generated in $Q \tilde{A}$ by $q \tilde{\mathbf{C}}$, the kernel of the folding map (4.5). In the analytic situation, in any of the completions $Q_{r} \tilde{A}$ we consider the closed ideal $I_{r}$, which is the completion of the ideal $I$ in the topology defined by the norm \|\|$_{Q_{r}}$. Thus we deduce that there are the following continuous isomorphisms

$$
\mathbf{C} \oplus Q_{r} A=Q_{r} \tilde{A} / I_{r}, \quad Q_{r} A=\bar{Q}_{r} \tilde{A} / I_{r}
$$

This observation, together with the properties of the family $\left\{\phi_{t}\right\}$ of homomorphisms of $Q_{\epsilon} \tilde{A}$ allows us to define a lift of $Q_{\epsilon} A$ back into $Q_{\epsilon} \tilde{A}$. In fact we prove 
4.7 Proposition. We have the following continuous isomorphism of locally convex superalgebras

$$
\phi_{1 / 2}\left(\bar{Q}_{\epsilon} \tilde{A}\right) \simeq Q_{\epsilon} A
$$

Proof. First let us note that $\phi_{1 / 2}(f)=\phi_{1 / 2}\left(f^{\gamma}\right)$ from which it follows that also $\phi_{1 / 2}(u)=\phi_{1 / 2}\left(u^{\gamma}\right)$. Thus if we apply the family $\phi_{t}$ to the superalgebra $\hat{Q}_{\epsilon} \tilde{A}$ then the effect of this action, when we let $t$ vary between 0 and $1 / 2$, is that it continuously deforms the subalgebra $\hat{Q} \tilde{\mathbf{C}}$ to $\mathbf{C}$. Indeed, we see that $\phi_{1 / 2}(L)=0$ and $\phi_{1 / 2}(W)=1$. Thus from the purely algebraic point of view $\phi_{1 / 2}(\hat{Q} \tilde{A})$ is the augmented free product $\mathbf{C} \oplus \phi_{1 / 2}(A) * \phi_{1 / 2}(A)$. From the universal properties of the algebras involved follows that the free product $\phi_{1 / 2}(A) * \phi_{1 / 2}(A)$ is isomorphic to $\hat{Q} A$. We now need to adapt this situation to our analytic context.

First we define a map $\phi_{1 / 2}\left(\bar{Q}_{\epsilon} \tilde{A}\right) \rightarrow Q_{\epsilon} A$. Since $\phi_{t}: \bar{Q}_{\epsilon} \tilde{A} \rightarrow \bar{Q}_{\epsilon} \tilde{A}$ is a continuous homomorphism for any $t \in[0,1]$, there exists a positive real $r$ such that $\phi_{1 / 2}(\bar{Q} \tilde{A}) \subset$ $\bar{Q}_{r} \tilde{A}$. We then put for any $\omega \in \bar{Q}_{\epsilon} \tilde{A}$

$$
\phi_{1 / 2}(\omega) \mapsto\left[\phi_{1 / 2}(\omega)\right] \in \bar{Q}_{r} \tilde{A} / I_{r}
$$

Since this map is just the restriction of the canonical projection $\bar{Q}_{r} \tilde{A} \rightarrow \bar{Q}_{r} \tilde{A} / I_{r}$ to the image of $\phi_{1 / 2}$, it is continuous.

To define the inverse map, let $[\omega] \in \bar{Q}_{r} \tilde{A} / I_{r}$ and let us choose a representative $\omega_{0} \in Q_{r} A$ of this class. We define

$$
\xi: \bar{Q}_{r} \tilde{A} / I_{r} \ni[\omega] \mapsto \phi_{1 / 2}\left(\omega_{0}\right) \in Q_{r^{\prime}} \tilde{A}
$$

This map is well defined, since for any $r>0, \phi_{1 / 2}$ vanishes on the ideal $I_{r}$, so that the above assignment does not depend on the choice of $\omega_{0}$. To show that it is continuous we note that, as the space $\bar{Q}_{r} \tilde{A} / I_{r}$ is equipped with the quotient space norm

$$
\|[\omega]\|_{Q_{r}}=\inf _{\eta \in I_{r}}\|\omega+\eta\|_{Q_{r}},
$$

for any $r$ there exists $\omega_{0} \in[\omega]$ such that $\left\|\omega_{0}\right\|_{Q_{r}} \leq 2\|[\omega]\|_{Q_{r}}$. Since $\phi_{t}$ is continuous for any $t \in[0,1]$, from the estimate (4.4) it now follows that there is an $r^{\prime}<r$ such that

$$
\|\xi([\omega])\|_{Q_{r^{\prime}}}=\left\|\phi_{1 / 2}\left(\omega_{0}\right)\right\|_{Q_{r^{\prime}}} \leq M\left\|\omega_{0}\right\|_{Q_{r}} \leq 2 M\|[\omega]\|_{Q_{r}}
$$

This shows that the map $\xi$ is continuous for all $r$ and so it is continuous in the locally convex topology on the union of $\bar{Q}_{r} \tilde{A} / I_{r} \simeq Q_{r} A$. This finishes the proof.

With this proposition we have constructed the following homotopy

$$
Q_{\epsilon} \tilde{A} \longrightarrow \mathbf{C} \oplus \phi_{1 / 2}\left(\bar{Q}_{\epsilon} \tilde{A}\right) \simeq \mathbf{C} \oplus Q_{\epsilon} A \stackrel{l}{\hookrightarrow} Q_{\epsilon} \tilde{A}
$$

where the map $l$ is the lift provided by the previous theorem. There is a corresponding homotopy of the reduced part $\bar{Q}_{\epsilon} \tilde{A}$ onto $Q_{\epsilon} A$

$$
\bar{Q}_{\epsilon} \tilde{A} \longrightarrow \phi_{1 / 2}\left(\bar{Q}_{\epsilon} \tilde{A}\right) \simeq Q_{\epsilon} A \stackrel{l}{\hookrightarrow} \bar{Q}_{\epsilon} \tilde{A}
$$


This homotopy will be a key ingredient when we compare the entire cyclic cohomology of $\bar{Q}_{\epsilon} \tilde{A}$ with the entire cyclic cohomology of $Q_{\epsilon} A$.

\section{Continuous supertraces on the Cuntz algebra and the entire cyclic cohomology}

In this section we shall describe supertraces on the Cuntz algebra and the notion of homotopy of supertraces. In our discussion we shall frequently use the identification of the Cuntz algebra $Q A$ with the space of differential forms $\Omega A$ equipped with the Fedosov product.

A cochain on $\Omega A$ is a linear functional $f$ with values in $\mathbf{C}$. Any cochain is uniquely determined by its components

$$
f_{n}\left(a_{0}, a_{1}, \cdots, a_{n}\right)=f\left(a_{0} d a_{1} \cdots d a_{n}\right)
$$

where $f_{n}: \Omega^{n} A \rightarrow \mathbf{C}$. Cochains defined by the above formula are called simplicially normalized since they satisfy $f_{n}\left(a_{0}, a_{1}, \ldots, a_{n}\right)=0$ if $a_{i}=1$ for some $i \geq 1$. The space of all cochains is a $\mathbf{Z} / 2$-graded complex $\Omega A^{*}$ equipped with the differential $b+B$, which is the transpose of the differential introduced on $\Omega A$. A cochain $f \in \Omega A^{*}$ is called harmonic if it is invariant under the action of $P$, the operator of spectral projection. If $f$ is a cocycle in the $b+B$ complex then $f b B=f(b+B) B=0$. Recall now the following fact [CuQ2, Propn. 8.5].

5.1 Proposition. If $f b B=0$ then $f$ is harmonic, i.e. $f P=f$, if and only if $f$ is $\kappa$-invariant, where $\kappa$ is the Karoubi operator introduced in Section 2

It follows that a cocycle is harmonic if and only if it is invariant under the action of the Karoubi operator $\kappa$.

If $A$ is a Banach algebra, the cochain complex $\Omega A^{*}$ contains the simplicially normalized entire cochain complex $\Omega_{\epsilon} A^{*}$, which by definition is a subcomplex consisting of all cochains satisfying the entire growth condition (1.1). We denote the cohomology of this complex by $H E_{s n}^{*}$ and call it simplicially normalized entire cyclic cohomology.

5.2 Remark. In the preprint version of their paper [CuQ2] Cuntz and Quillen proved that $H E_{s n}^{*}(A)=H^{*}\left(\left(P \Omega_{\epsilon} A\right)^{*}, b+B\right)$.

A supertrace on the Cuntz algebra $Q A$ is a linear functional $\tau$ which satisfies the following identity

$$
\tau(\omega \eta)=(-1)^{|\omega||\eta|} \tau(\eta \omega)
$$

for any two homogeneous elements $\omega$ and $\eta$ of $Q A$.

Since the Cuntz algebra is a superalgebra, any functional $f$ on $Q A$ is uniquely represented as the sum of even and odd components. By definition, a cochain is even if $f \gamma=f$ and is odd when $f \gamma=-f$ where $\gamma$ is the canonical automorphism of $Q A$. 
If we identify the Cuntz algebra with the algebra of differential forms equipped with the Fedosov product then we can relate supertraces with cocycles in the complex $(\Omega A, b+B)$. And so a cochain $\tau$ is a supertrace if and only if

$$
\tau_{n-1} b=2 \tau_{n+1} d
$$

and $\tau_{n} \kappa=\tau_{n}$, for $n>0$. Let

$$
z_{2 m}=\frac{(-1)^{m}}{m !}, \quad z_{2 m+1}=\frac{(-1)^{m} 2^{m}}{(2 m+1) ! !}
$$

and put $\tau^{z}=\tau z$. When we use that $\tau_{n} B=n \tau_{n} d$ when $\tau$ is $\kappa$-invariant, then we derive instantly the following property satisfied by the rescaled cochains:

$$
\tau_{n-1}^{z} b+\tau_{n+1}^{z} B=0
$$

for all positive $n$. In other words we have the following [CuQ2, Cor. 8.2].

5.3 Proposition. A linear functional $\tau$ on $\Omega A$ is a supertrace on $Q A$ if and only if the rescaled cochain $\tau^{z}$ satisfies the identities $\tau^{z}(b+B)=0$ and $\tau^{z}=\tau^{z} \kappa$ in positive degrees.

Let us now consider the cases where $\tau$ is an even or an odd supertrace. In the even case there is clearly an equivalence between even supertraces on $Q A$ and harmonic cocycles. In the odd case the situation is slightly different since now the cocycle condition in the lowest degree is equivalent to

$$
\left(\tau^{z}(b+B)\right)_{0}(a)=\left(\tau^{z} B\right)_{1}(d a)=\tau^{z}(d a)=0
$$

Thus $\tau^{z}$ is a cocycle if and only if $\tau^{z} d a=0$ for any $a \in A$. The same condition expressed in terms of the Cuntz algebra reads $\tau^{z}(q(a))=0$ for any $a \in A$. Thus odd harmonic cocycles correspond to odd traces $\tau$ on $Q A$ for which $\tau^{z}(q(a))=0$ for all $a \in A$. To summarise we state the following.

\subsection{Proposition.}

1. There is a bijection (given by the family $z$ of constants introduced above) between the even harmonic cocycles in $\left(P \Omega_{\epsilon} A^{*}, b+B\right)$ and continuous even supertraces on $Q_{\epsilon} A$.

2. There is a bijection between the odd harmonic cocycles in $P \Omega_{\epsilon} A$ and odd continuous supertraces $\tau$ on $Q_{\epsilon} A$ satisfying the condition $\tau(q(a))=0$, for all $a \in A$.

Another crucial ingredient of our reasoning is the notion of homotopy of supertraces. To describe homotopy of supertraces on $Q A$ we use the $X$-complex approach of Cuntz and Quillen [CuQ3]. We therefore need to discuss supertraces on the superbimodule $\Omega^{1} Q A$ over the Cuntz algebra $Q A$. As before, supertraces on 
$\Omega^{1} Q A$ are linear functionals $T$ which vanish on the supercommutator quotient space $\Omega^{1} Q A_{\natural s}=\Omega^{1} Q A /\left[Q A, \Omega^{1} Q A\right]_{s}$. First of all, it turns out that we may identify the supercommutator quotient space $\Omega^{1} Q_{\natural s}$ with the space $\Omega^{1} A \oplus \Omega A$ [CuQ4]. We shall use the following notation. Let $\theta$ be the inclusion $\theta: A \rightarrow Q A$ and $\theta^{\gamma}$ the composition of $\theta$ with the canonical automorphism $\gamma$ of $Q$. We let $p$ denote the cochain on $A$ given by $p=\left(\theta+\theta^{\gamma}\right) / 2$ and similarly $q=\left(\theta-\theta^{\gamma}\right) / 2$. We then have the following identification of the even part $\left(\Omega^{1} Q_{\natural s}\right)^{e v}$ of the supercommutator space

$$
\bigoplus_{n \geq 0} A \otimes \bar{A}^{2 n+1} \stackrel{\simeq}{\longrightarrow}\left(\Omega^{1} Q_{\natural s}\right)^{e v}
$$

This isomorphism is given by the cochains $\left(\theta^{\gamma} q^{2 n} d \theta\right)^{e v}$. The odd part of the supercommutator space is

$$
\Omega^{1} A \oplus \bigoplus_{n \geq 1} A \otimes \bar{A}^{\otimes 2 n} \stackrel{\simeq}{\longrightarrow}\left(\Omega^{1} Q A_{\natural s}\right)^{o d}
$$

where this time the isomorphism is given by cochains $(\theta d \theta)^{o d},\left(\theta q^{2 n-1} d \theta\right)^{o d}$. Using this coordinate system, Cuntz and Quillen show [CuQ4] that the components of an even supertrace $T$ on $\Omega^{1} Q$ are given by the following cochains

$$
T_{2 n+1}=T\left(\theta^{\gamma} q^{2 n} d \theta\right): A \otimes \bar{A}^{2 n} \rightarrow \mathbf{C}
$$

On the other hand, an odd supertrace $T$ has the following cochains as its components

$$
T_{2 n}=T\left(\theta q^{2 n-1} d \theta\right), \quad T_{1}=T \theta d \theta, \quad T_{1} b=0
$$

To summarise, using the above isomorphisms, we have can represent any supertrace $T$ on $\Omega^{1} Q$ in terms of cochains on $\Omega^{1} A \oplus \Omega A$. From now on components of functionals on $\Omega^{1} Q A$ will always be written in terms of these isomorphisms. We can now state the following $[\mathrm{CuQ} 4]$

5.5 Proposition. Let $T$ be a supertrace on $\Omega^{1} Q A$ and let $\delta$ be the differential $\delta: Q A \rightarrow \Omega^{1} Q A$. Then we have

$$
\begin{array}{llc}
(T \circ \delta)_{2 n} & = & -n P b T_{2 n-1}+B T_{2 n+1} \\
(T \circ \delta)_{2 n+1} & = & (n+1 / 2) P b T_{2 n}-B T_{2 n+2}
\end{array}
$$

We say that two supertraces on $Q A$ are homotopic if and only if there exists a supertrace $T$ on $\Omega^{1} Q A$ such that

$$
\tau_{0}-\tau_{1}=T \circ \delta
$$

5.7 Proposition. If $\tau_{0}$ and $\tau_{1}$ are homotopic supertraces on $Q A$ then the corresponding cocycles $\tau_{0}^{z}$ and $\tau_{1}^{z}$ are cohomologous in the cochain complex $\left(\Omega A^{*}, b+B\right)$. 
Proof. Let us first assume that $\tau_{0}$ and $\tau_{1}$ are even homotopic supertraces on $Q A$. Since the difference of two even supertraces is an even supertrace, we may use the rescaling given by the family $z$ of constants to write the formula for the corresponding cocycle on $\Omega A$ equipped with the differential $b+B$. Using the formula (5.6) we have

$$
\begin{aligned}
\left(\tau_{1}-\tau_{0}\right)_{2 n}^{z} & =\frac{(-1)^{n}}{n !}\left(\tau_{1}-\tau_{0}\right) \\
& =\frac{(-1)^{n}}{n !}(T \circ \delta)_{2 n} \\
& =\frac{(-1)^{n}}{n !}\left(-n P b T_{2 n-1}+B T_{2 n+1}\right)
\end{aligned}
$$

The rescaled cochain $\left(\tau_{1}-\tau_{0}\right)^{z}$ is a $\kappa$-invariant cocycle in the complex $(\Omega A, b+B)$, and so it follows from proposition 5.1 that it is invariant under the operator $P$. The above identity indicates that therefore the right hand side must also be $P$-invariant, and so we have

$$
\left(\tau_{1}-\tau_{0}\right)^{z}=\left(\tau_{1}-\tau_{0}\right)^{z} P=-n b P T_{2 n-1}+B P T_{2 n+1}
$$

where we have used that $P$ commutes with $b$ and $B$. If we denote by $\tilde{T}_{2 n+1}=$ $(-1)^{n} P T_{2 n+1} / n$ ! we may write

$$
\left(\tau_{1}-\tau_{0}\right)_{2 n}^{z}=b \tilde{T}_{2 n-1}+B \tilde{T}_{2 n+1}
$$

which demonstrates that the cocycle $\left(\tau_{1}-\tau_{0}\right)^{z}$ is a coboundary.

Let us now assume that $\tau_{0}$ and $\tau_{1}$ are odd supertraces. In this case, the rescaled cocycle $\left(\tau_{1}-\tau_{0}\right)^{z}$ satisfies the following homotopy identity

$$
\begin{aligned}
\left(\tau_{1}-\tau_{0}\right)_{2 n+1}^{z} & =\frac{(-1)^{n} 2^{n}}{(2 n+1) ! !}\left(\left(n+\frac{1}{2}\right) P b T_{2 n}-B T_{2 n+2}\right) \\
& =b \tilde{T}_{2} n+B \tilde{T}_{2 n+2}
\end{aligned}
$$

where $\tilde{T}_{2 n}=\frac{(-1)^{n} 2^{n}}{(2 n+1) ! !} P T$. We have used again the fact that the cocycle $\left(\tau_{1}-\tau_{0}\right)^{z}$ is $P$-invariant.

Finally, in the case when $A$ is a Banach algebra, one shows as in [Co2, Prop. 2.4] that cocycles coming from a continuous supertrace on $Q_{\epsilon} A$ satisfy the entire growth condition.

Our next step is to prove that the family $\phi_{t}$ of homomorphisms establishes an isomorphism between the homotopy classes of supertraces on $\bar{Q}_{\epsilon} \tilde{A}$ and $Q_{\epsilon} A$.

5.8 Proposition. Let $\tau$ be a continuous supertrace on $\bar{Q}_{\epsilon} \tilde{A}$. Then $\tau_{t}=\tau \circ \phi_{t}$ is a continuous supertrace on $\bar{Q}_{\epsilon} \tilde{A}$ whose homotopy class does not depend on $t$. 
Proof. Since $\phi_{t}$ is a superalgebra homomorphism, it is clear that $\tau_{t}=\tau \circ \phi_{t}$ is a continuous supertrace for all $t \in[0,1]$. Let us define the following map $T$ on $\Omega^{1} Q_{\epsilon} \tilde{A}$. For any $\omega, \eta$ in $\bar{Q}_{\epsilon} \tilde{A}$ we put

$$
T(\omega \delta \eta)=\int_{0}^{1} \tau\left(\phi_{t}(\omega) \dot{\phi}_{t}(\eta)\right) d t
$$

We claim that $T$ is a well-defined continuous supertrace on $\Omega^{1} Q_{\epsilon} \tilde{A}$. Let us put aside for the moment the problem of differentiability of $\phi_{t}$, and concentrate on the purely algebraic supertrace condition.

If $\alpha \in \bar{Q}_{\epsilon} \tilde{A}$ and $\omega, \eta$ are as above, then the supercommutator $[\alpha, \omega \delta \eta]_{s}$ is given by

$$
\begin{aligned}
{[\alpha, \omega \delta \eta]_{s} } & =\alpha \omega \delta \eta-(-1)^{|\alpha||\omega||\eta|} \omega \delta \eta \alpha \\
& =\alpha \omega \delta \eta-(-1)^{|\alpha||\omega||\eta|}(\omega \delta(\eta \alpha)-\omega \eta \delta \alpha)
\end{aligned}
$$

Using the fact that $\tau$ is a supertrace on $\bar{Q}_{\epsilon} \tilde{A}$ we have for any $t \in[0,1]$

$$
\begin{aligned}
\tau\left(\phi_{t}(\omega) \dot{\phi}_{t}(\eta \alpha)\right)= & \tau\left(\phi_{t}(\omega) \dot{\phi}_{t}(\eta) \phi_{t}(\alpha)\right) \\
& +\tau\left(\phi_{t}(\omega) \phi_{t}(\eta) \dot{\phi}_{t}(\alpha)\right) \\
= & (-1)^{|\alpha||\omega||\eta|} \tau\left(\phi_{t}(\alpha) \phi_{t}(\omega) \dot{\phi}_{t}(\eta)\right) \\
& +\tau\left(\phi_{t}(\omega) \phi_{t}(\eta) \dot{\phi}_{t}(\alpha)\right.
\end{aligned}
$$

we see that applying $T$ to both sides of (5.9) shows that $T$ vanishes on supercommutators and so it is a supertrace.

We now need to check that we can differentiate and integrate $\phi_{t}$ in the way required by the above definition of the supertrace $T$. First of all, from the definition (4.1) it follows that $\dot{a}_{t}=\dot{\phi}_{t}(a)=W_{-t}[L, a] W_{t}$, for any $a \in A$, since $L$ commutes with $W_{t}=e^{t L / 2}$. Similarly, we have that $\dot{a}_{t}^{\gamma}=e^{-t L / 2}\left[a^{\gamma}, L\right] e^{t L / 2}$. Using this observation we have

$$
\begin{aligned}
& \dot{\phi}_{t}(p(a))=p\left(\left[L, a_{t}\right]\right) \\
& \dot{\phi}_{t}(q(a))=q\left(\left[L, a_{t}\right]\right)
\end{aligned}
$$

We can now find estimates for the norms of these elements with respect to the norm in the algebra $Q_{r} \tilde{A}$. Using the two formulae above together with our previous estimates we see that for all $r<r_{0}$

$$
\begin{aligned}
\left\|p\left(\left[L, a_{t}\right]\right)\right\|_{Q_{r}} & \leq 2 C_{r}\|L\|_{Q_{r}}\left\|q\left(a_{t}\right)\right\|_{Q_{r}} \\
& \leq 2 C_{r}\|L\|_{Q_{r}} M\|q(a)\|_{Q_{r}} \\
& =2 C_{r} r\|L\|_{Q_{r}} M\|p(a)\|_{Q_{r}}
\end{aligned}
$$


where, as usual, $C_{r}=1+r^{2}$. Moreover, using the estimate (3.4) we have

$$
\begin{aligned}
\left\|q\left(\left[L, a_{t}\right]\right)\right\|_{Q_{r}} & \leq 2 C_{r}\|L\|_{Q_{r}}\left\|p\left(a_{t}\right)\right\|_{Q_{r}} \\
& \leq 2 C_{r} M N r\|p(a)\|_{Q_{r}} \\
& =2 C_{r} M N\|q(a)\|_{Q_{r}}
\end{aligned}
$$

It follows that if $\omega_{n}=p\left(a_{0}\right) q\left(a_{1}\right) \cdots q\left(a_{n}\right)$ then

$$
\begin{aligned}
\left\|\left(\dot{\omega}_{n, t}\right)\right\|_{Q_{r}} & =\left\|\dot{p}_{t}\left(a_{0}\right) q_{t}\left(a_{1}\right) \cdots q_{t}\left(a_{n}\right)+\sum_{i} p_{t}\left(a_{0}\right) q_{t}\left(a_{1}\right) \cdots \dot{q}_{t}\left(a_{i}\right) \cdots q_{t}\left(a_{n}\right)\right\|_{Q_{r}} \\
& \leq 2 C_{r}\left(\|L\|_{Q_{r}} r+N\right) M^{n+1}\left\|\omega_{n}\right\|_{Q_{r}}
\end{aligned}
$$

Let us put $s=2 C_{r}\left(\|L\|_{Q_{r}} r+N\right)$. We then have in general that if $\omega=\sum \omega_{n} \in Q_{r^{\prime}} \tilde{A}$ then

$$
\left\|\dot{\phi}_{t}(\omega)\right\|_{Q_{r}} \leq \sum_{n \geq 0} s M^{n+1} r\left\|\omega_{n}\right\|_{Q_{r}}
$$

and the series on the right hand side is convergent for $r<r^{\prime} / M$.

It is slightly easier to show that $\phi_{t}$ behaves well with respect to integration. We have

$$
\left\|\int_{0}^{1} \phi_{t}(\omega) d t\right\|_{Q_{r}} \leq \int_{0}^{1}\left\|\phi_{t}(\omega)\right\|_{Q_{r}} d t \leq \int_{0}^{1} M\|\omega\|_{Q_{r}} d t \leq M\|\omega\|_{Q_{r}}
$$

The above results show that indeed the homotopy class of the supertrace $\tau_{t}=$ $\tau \circ \phi_{t}$ does not depend on $t$. Since we want to use supertraces to describe entire cyclic cocycles, we must also check that if $\tau$ is an odd supertrace on $\bar{Q}_{\epsilon} \tilde{A}$ such that $\tau(q(a))=0$ for any $a$ that $\tau_{t}$ has the same property. But this follows directly from the trace identity

$$
\tau \circ \phi_{t} q(a)=(1 / 2) \tau\left(a_{t}-a_{t}^{\gamma}\right)=(1 / 2) \tau\left(a-a^{\gamma}\right)=\tau q(a)=0
$$

This finishes the proof.

5.10 Corollary. The family $\phi_{t}$ of homomorphisms of $Q_{\epsilon} \tilde{A}$ induces an isomorphism of homotopy classes of supertraces on $\bar{Q}_{\epsilon} \tilde{A}$ and $Q_{\epsilon} A$.

\section{Simplicial normalization in the entire cyclic cohomology}

In his definition of entire cyclic cohomology, Connes [Co2] uses the complex $\left(C(A)^{*}, b+B\right)$, whose elements are simplicially unnormalized cochains described in the introduction. By definition, the entire cochain complex is the subcomplex $C_{\epsilon}(A)^{*}$ of cochains satisfying the entire growth condition (1.1). Let us denote by $H E^{*}(A)$ the cohomology of this complex, which we shall call the entire cyclic cohomology.

Inside the entire complex $C_{\epsilon}(A)^{*}$ there is a subcomplex of simplicially normalized entire cochains $\left(\Omega_{\epsilon}(A)^{*}, b+B\right)$. We shall call the cohomology of this complex the simplicially normalized entire cohomology which we shall denote by $H E_{s n}^{*}(A)$. Our main goal in this paper is to prove the following simplicial normalization theorem. 
6.1 TheOREM. The inclusion

$$
\Omega_{\epsilon} A^{*} \rightarrow C_{\epsilon}(A)^{*}
$$

induces an isomorphism

$$
H E_{s n}^{*}(A) \simeq H E^{*}(A)
$$

Proof. A starting point for the proof of this theorem is the following theorem proved by Connes [Co2, Lemma 1.6, Propn. 2.3 and 2.4]. First we say that a cochain $\psi \in C(A)^{*}$ is normalized in the sense of Connes (or simply normalized) if and only if $\psi B_{0}(1-\lambda)=0$, where $B_{0}=(1-\lambda) h$ and $h$ is any operator of degree +1 such that $\left[b^{\prime}, h\right]=1$.

\subsection{ThEOREM.}

1. Any entire cyclic cocycle is cohomologous to a normalized entire cocycle.

2. There is a 1-1 correspondence between even normalized entire cocycles and continuous supertraces on $\bar{Q}_{\epsilon} \tilde{A}$ given by $\psi \mapsto\left(\psi, B_{0} \psi\right)$.

Moreover, there is the following commutative diagram of $\mathbf{Z} / 2$-graded chain complexes equipped with the differential $b+B$.

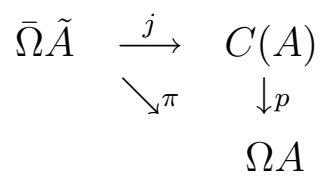

where the map $j: \bar{\Omega} \tilde{A} \rightarrow C(A)$ is given by $j(\omega, \eta)=\omega+B_{0} \eta$. The map $\pi$ : $\bar{\Omega} \tilde{A} \rightarrow \Omega A$ is induced by the canonical projection $p: \bigoplus_{n \geq 0} A \otimes \bar{A}^{\otimes n} \rightarrow \Omega A$ given by $p_{n}\left(a_{0}, a_{1}, \ldots, a_{n}\right)=a_{0} d a_{1} \cdots d a_{n}$. When elements of $\bar{\Omega} \tilde{A}$ are represented by column vectors $\left(\begin{array}{c}x \\ y\end{array}\right) \in A^{\otimes n+1} \oplus A^{\otimes n}$ then the projection $\pi$ is given by $\pi_{n}=\left(p_{n}, d p_{n-1}\right)$.

Let us assume that $f$ is a cocycle in $\Omega_{\epsilon} A$. Following Remark 5.2 we may assume that $f$ is harmonic. Then it is also $\kappa$-invariant, so there is an associated supertrace $\tau=f z^{-1}$ on $Q A$. The image of $f$ in $\bar{\Omega} \tilde{A}$ is $(f p, f d p)$, which is the same as $\left(f p, f B_{0} p\right)$, since $p B_{0}=d p$. Therefore, this cocycle corresponds to a supertrace on $\bar{Q} \tilde{A}$ and so descends to a normalized cocycle $f p$ in $C_{\epsilon}(A)^{*}$. But this proves that the image $f p$ of the cocycle $f$ is a normalized cocycle. It follows that the map $H E_{s n}^{*}(A) \rightarrow H E^{*}(A)$ is injective.

Let us now take a normalized cocycle $g \in C_{\epsilon}(A)^{*}$. This cocycle lifts to a supertrace $\tau$ on $\bar{Q}_{\epsilon} \tilde{A}$, which in coordinates is given by $\left(g z^{-1}, g B_{0} z^{-1}\right)$. Corollary 5.10 implies that $\tau$ is homotopic to a supertrace of the form $\tau^{\prime} \pi$, where $\tau^{\prime}$ is a supertrace on $Q_{\epsilon} A$. To be precise, $\tau^{\prime}=\tau \circ l$. It is clear that in our usual coordinates on $\bar{\Omega}_{\epsilon} \tilde{A}$, this supertrace corresponds to the cocycle

$$
\left(\tau^{\prime} \pi\right)^{z}=\left(\left(\tau^{\prime} p\right)^{z}, \quad\left(\tau^{\prime} d p\right)^{z}\right)=\left(\left(\tau^{\prime} p\right)^{z}, \quad\left(\tau^{\prime} p B_{0}\right)^{z}\right)
$$


This cocycle descends to a normalized cocycle $\left(\tau^{\prime} p\right)^{z}$ in $C_{\epsilon}(A)^{*}$. Since $\tau$ is homotopic to $\tau^{\prime} \pi$ the cocycle $\tau^{z}$ is cohomologous to the cocycle $\left(\tau^{\prime} \pi\right)^{z}$. It follows that the cocycles $g$ and $\left(\tau^{\prime} p\right)^{z}$ are cohomologous in $C_{\epsilon}(A)^{*}$. On the other hand, the supertrace $\tau^{\prime}$ determines a $\kappa$-invariant cocycle (which is also $P$-invariant) cocycle $\left(\tau^{\prime}\right)^{z}$ in $\Omega_{\epsilon} A^{*}$. But it is clear that the canonical inclusion $\Omega_{\epsilon} A^{*} \rightarrow C_{\epsilon}(A)^{*}$ sends $\left(\tau^{\prime}\right)^{z}$ to $\left(\tau^{\prime} p\right)^{z}$. This shows that

$$
H E_{s n}^{*}(A) \ni\left[\left(\tau^{\prime}\right)^{z}\right] \mapsto\left[\left(\tau^{\prime} p\right)^{z}\right]=[g] \in H E^{*}(A)
$$

It follows that the induced map $H E_{s n}^{*}(A) \rightarrow H E^{*}(A)$ is surjective.

6.4 REMARK. It is an amusing application of this theorem to calculate the entire cyclic cohomology of $\mathbf{C}$. Since $Q \mathbf{C}=\mathbf{C}$ in degree zero and zero otherwise, we have that $H E^{\text {even }}(\mathbf{C})=\mathbf{C}$, since supertraces on $\mathbf{C}$ are just linear maps $\mathbf{C} \rightarrow \mathbf{C}$, and $H E^{o d d}(\mathbf{C})=0$.

\section{Homotopy invariance of the entire cyclic cohomology}

As it was mentioned in the introduction, the use of supertraces on the Cuntz algebra is a very useful tool in studying entire cyclic cohomology. In this section we present another example of such an application in the case of homotopy invariance of this cohomology theory $[\mathrm{K}]$.

Let us then consider two unital Banach algebras $A$ and $B$ and a continuous family of homomorphisms $f_{t}: A \rightarrow B$. We assume that each of the homomorphisms $f_{t}$ is continuous for $t \in[0,1]$ and that the family is uniformly bounded, i.e. that there exists a constant $M$ such that $\left\|f_{t}\right\| \leq M$ for $t \in[0,1]$. We suppose, moreover, that the corresponding family of derivatives $\dot{f}_{t}$ with respect to the parameter $t$ is continuous and uniformly bounded by a constant $N$ on the interval $[0,1]$. We then have the following.

7.1 Theorem. Let $f_{t}: A \rightarrow B$ be a family of homomorphisms of Banach algebras satisfying the properties given above. Then $f_{0}$ and $f_{1}$ induce the same map on the entire cyclic cohomology $H E^{*}(B) \rightarrow H E^{*}(A)$.

Proof. The family of homomorphisms $f_{t}: A \rightarrow B$ extends to a family of continuous homomorphisms of continuous homomorphisms of the corresponding Cuntz algebras $\Phi_{t}: Q_{r} A \rightarrow Q_{r} B$ for any positive real $r$ if we define

$$
\Phi_{t}\left(p\left(a_{0}\right) q\left(a_{1}\right) \cdots q\left(a_{n}\right)\right)=p\left(f_{t}\left(a_{0}\right)\right) q\left(f_{t}\left(a_{1}\right)\right) \cdots q\left(f_{t}\left(a_{n}\right)\right)
$$

To prove that this map is continuous for any positive real $r$ we first need to find estimates for $\left\|p\left(f_{t}(a)\right)\right\|_{Q_{r}}$ and $\left\|q\left(f_{t}(a)\right)\right\|_{Q_{r}}$. From the definition of the norm on the Cuntz algebra we have

$$
\left\|p\left(f_{t}(a)\right)\right\|=\left\|f_{t}(a)\right\| \leq M\|a\|=M\|p(a)\|
$$


and

$$
\begin{aligned}
\left\|q\left(f_{t}(a)\right)\right\| & =\left\|d\left(f_{t}(a)\right)\right\|=\inf _{\lambda \in \mathbf{C}}\left\|f_{t}(a)+\lambda f_{t}(1)\right\| \\
& \leq\left\|f_{t}\right\| \inf _{\lambda \in \mathbf{C}}\|a+\lambda\|=\left\|f_{t}\right\|\|d a\| \\
& \leq M\|q(a)\|
\end{aligned}
$$

Thus if $\omega=\sum \omega_{n} \in \Omega_{r} A$ then using the above estimates we have that

$$
\begin{aligned}
\|\omega\|_{Q_{r}} & =\sum\left\|\Phi_{t}\left(\omega_{n}\right)\right\| r^{n} \leq M^{n+1}\left\|\omega_{n}\right\| r^{n} \\
& =M\|\omega\|_{Q_{r}}
\end{aligned}
$$

This shows that $\Phi_{t}$ is a continuous homomorphism $\Phi_{t}: Q_{r} A \rightarrow Q_{r} B$ for all positive $r$ and so it extends to a continuous homomorphism $\Phi_{t}: Q_{\epsilon} A \rightarrow Q_{\epsilon} B$. This means that $\Phi_{t}$ induces a map $\Phi_{t}^{*}$ from the space of continuous supertraces on $Q_{\epsilon} B$ to the space of continuous supertraces on $Q_{\epsilon} A$.

We now want to prove that $f_{0}$ and $f_{1}$ induce the same map on the entire cyclic cohomology. Let us then assume that $T$ is a continuous supertrace on $Q_{\epsilon} B$, we want to show that the supertraces on $Q_{\epsilon} A T \circ \Phi_{0}$ and $T \circ \Phi_{1}$ are homotopic. But we can define a supertrace on $\Omega^{1} Q_{\epsilon} B$ by the formula

$$
\tau(\omega \delta \eta)=\int_{0}^{1} T\left(\Phi_{t}(\omega) \dot{\Phi}_{t}(\eta)\right) d t
$$

It is clear that we have $T \delta \eta=T \Phi_{1}(\eta)-T \Phi_{0}(\eta)$ which means that the supertraces $T \Phi_{0}$ and $T \Phi_{1}$ are homotopic. We now need to check that $\Phi_{t}$ and $\dot{\Phi}_{t}$ are continuous. But since we have for any $a \in A$

$$
\begin{aligned}
& d / d t\left(p\left(f_{t}(a)\right)\right)=p\left(\dot{f}_{t}(a)\right) \\
& d / d t\left(q\left(f_{t}(a)\right)\right)=q\left(\dot{f}_{t}(a)\right)
\end{aligned}
$$

we can estimate the norms of these elements as before, using the fact that the derivative $\dot{f}_{t}$ is uniformly bounded by $N$. We find that

$$
\begin{aligned}
& \left\|p\left(\dot{f}_{t}(a)\right)\right\| \leq N\|p(a)\| \\
& \left\|q\left(\dot{f}_{t}(a)\right)\right\| \leq N\|q(a)\|
\end{aligned}
$$

¿From this point on the proof is the same as in the case of simplicial normalization theorem.

\section{Acknowledgements}

It is a pleasure to thank Alain Connes, Joachim Cuntz and Daniel Quillen for numerous discussions. In particular, the idea of the homotopy used in the proof of the main theorem is due to J. Cuntz. I am also very grateful to him for his kind hospitality in Heidelberg in June and December 1992, where some preliminary work was carried out. A major part of this work was done at the I.H.E.S., whose generous support I gratefully acknowledge. 


\section{References}

[Arv] W. Arveson, The harmonic analysis of automorphism groups, in: Proc. Symp. Pure Math., R. Kadison ed., 38(1), 199-270.

[B] J. Brodzki, Binomial identities in the Cuntz algebra of complex numbers, Expositiones Mathem. to appear.

[Co1] A. Connes, Non-commutative differential geometry, Publ. Math. I.H.E.S 62 (1985), 257-360.

[Co2] A. Connes, Entire cyclic cohomology of Banach algebras and characters of $\theta$-summable Fredholm modules, $K$-theory, 1 (1988), 519-548.

[Co3] A. Connes Non commutative geometry, preprint I.H.E.S.

[CC] A. Connes and J. Cuntz, Quasihomomorhismes, cohomologie cyqlique et positivité, Comm. Math. Phys. 114 (1988), 515-526.

[Cu1] J. Cuntz, A new look at KK-theory, K-theory 1, (1987), 31-51.

[Cu2] J. CunTz Quantized differential forms in non-commutative topology and geometry, Contemporary Mathematics 145 (1993), 65-78.

[CuQ1] J. Cuntz And D. Quillen, Algebra extensions and nonsingularity, to appear.

[CuQ2] J. Cuntz And D. Quillen, Operators on noncommutative differential forms and cyclic homology, to appear.

[CuQ3] J. Cuntz And D. Quillen, Cyclic homology and nonsingularity, to appear.

[CuQ4] J. Cuntz And D. Quillen, unpublished manuscript.

[Ka1] C. Kassel, Cyclic homology, comodules and mixed complexes, J. Alg. 107 (1987), 195-216.

[Ka2] C. Kassel, Homologie cyclique, caractère de Chern et lemme de perturbation, J. Reine Angew. Mathem. 408 (1990), 159-180.

[K] M. Khalkhali, On the entire cyclic cohomology of Banach algebras II, Homotopy Invariance, preprint Heidelberg 1992.

[LQ] J.L. LOdAY, D. Quillen, Cyclic homology and the Lie algebra of matrices, Comment. Math. Helvetici 59 (1984), 565-591.

[MacL] S. MacLane, Homology, Springer Verlag, Berlin-New York, (1975). 